Editorial

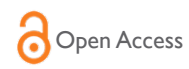

\title{
Editorial on rehabilitation and geriatrics
}

\section{Editorial}

I have the privilege of introducing the first volume of the International Physical Medicine \& Rehabilitation Journal with an editorial. Physical Medicine and Rehabilitation (PM\&R) is an extremely unique medical specialty: we physiatrists practice across widely diverse areas of the specialty such as general medical rehabilitation, musculoskeletal, neurological, pediatrics, pain, amputee care, geriatrics and oncological to name a few. $P M \& R$ is called a quality of life specialty; the specialty is well recognized for its focus on 'function' and emphases on quality of life. The practitioners of this specialty are no less diverse coming from various parts of the world and with many different cultural, racial, and religious beliefs; however, the common thread is that we treat people with disabilities. In my home institution, our guiding principles of patient care are based on the "ICARE" Values: I for Integrity, C for Compassion, A for Accountability, R for Respect and E for Excellence These are the principles for our training, practice, and teaching of our future medical students, residents, and Fellows. As physiatrists, the "ICARE" Values exemplify the approach we take with our patients who expect to be treated with dignity; and we owe it to them, and to each other.

Having trained in PM\&R as a resident after Internal Medicine, I found it fascinating that we specialize in treating a population that is clearly older with often complicated medical, psychological, social, and economic problems. This aspect sparked my interest to do a Fellowship in Geriatric rehabilitation, and I was fortunate to work with a world renown geriatrician Dr. Franklin Williams who instilled the importance of rehabilitation to geriatrics. I would like to focus on this topic as the aging of the US and world populations known as the 'silver tsunami' calls for an international attention to the exponential need for rehabilitation services in the coming years. By the year 2050, people over the age of 65 years will double to more than 80 million in the US. Similarly, according to the United Nations, from 2006 to 2030 the rate of growth of the aging population is expected to grow by $50 \%$ in the developed countries and $140 \%$ in developing counties.

The aging population is surviving longer and as the life expectancy is reaching new heights, so are the challenges of disease and disability in this age group. Chronic health problems with several co-morbidities are almost a norm in the aging older adults. In the United States alone it is reported that more than $45 \%$ of the older adults living in the community have one or more chronic conditions and hence multimorbidity is common in this age group. This in itself carries a higher risk for functional dependency. Advanced age is often accompanied by poor health with chronic disease and disability resulting in declining function in self-care and mobility. Physiatrists play a major role in contributing to improving this population's function by treating the co-morbidities, directing physical and occupational therapies and prescribing mobility aids and assistive technology.

There is a correlation between incidence of co-morbidities
Volume I Issue I - 2017

\author{
Rao Poduri K \\ Department of Physical Medicine and Rehabilitation, University \\ of Rochester Medical Center, USA
}

Correspondence: Rao Poduri K, Department of Physical Medicine and Rehabilitation, University of Rochester Medical Center, Rochester, NY, USA

Email KR_Poduri@URMC.Rochester.edu

Received: April 28, 2017 | Published: April 28, 2017

and economic deprivation. Older adults visit physicians twice as frequently as their younger counterparts and are hospitalized twice as often and stay $50 \%$ longer. The period of disability before death carries economic and societal challenges. Older adults take more medications and polypharmacy is identified as a risk in this vulnerable population. Disease, disability and dependence with added financial burden of care needs put this aging population at risk for social isolation, depression and institutionalization. Rehabilitation specialists can play significant roles in the lives of aging population in many ways.

Our ever expanding role includes caring for individuals with strokes, spinal cord and brain injuries, trauma musculoskeletal conditions with pain, cancer, debility etc...in the acute hospital, inpatient and outpatient levels, and in some situations even at home.

The overarching goal of our role is to facilitate function, maintenance of health by preventive measures and making the geriatric population age gracefully. No other specialty is equipped with our physiatric expertise in managing function and we make a great contribution in the care of these people for years to come. Nonetheless, our role does not end with caring for the geriatric population but also provides us with an opportunity to educate our colleagues literally in every specialty except Pediatrics.

I would recommend the rehabilitation community to take the challenge and add "Healthy functional life to the years of our geriatric patients than mere years to their lives with heroic and unnecessary interventions." I will end with a quote by Eleanor Roosevelt "The greatest tragedy of old age is the tendency for the old to feel unneeded, unwanted, and of no use to anyone; the secret of happiness in the declining years is to remain interested in life, as active as possible, useful to others, busy, and forward looking".

\section{Acknowledgements}

None

\section{Conflict of interest}

The author declares no conflict of interest. 